\title{
Accuracy improvement of collocation method by using the over-range collocation points for 2-D and 3-D problems
}

\author{
Yong-Ming GUO*, Kouji SHIOYA*, Kei OOBUCHI*, Genki YAGAWA** and Shunpei KAMITANI* \\ ${ }^{*}$ Graduate School of Science and Engineering, Kagoshima University \\ 1-21-40 Korimoto, Kagoshima City, 890-0065 Japan \\ E-mail: guoy@mech.kagoshima-u.ac.jp \\ **Tokyo University of Science \\ 2641 Yamazaki, Noda-shi, 278-8510 Japan
}

Received 21 November 2013

\begin{abstract}
It has been shown that the significance of the positivity conditions in the collocation methods (CM), and the violation of the positivity conditions can significantly result in a large error in the numerical solution. For boundary points, however, the positivity conditions cannot be satisfied, obviously. To overcome the demerit of the $\mathrm{CM}$, the over-range collocation method (ORCM) has been proposed. In the ORCM, some over-range collocation points are introduced which are located at the outside of the domain of an analyzed body, and at the over-range collocation points no satisfaction of any governing partial differential equation or boundary condition is needed. In this paper, it is shown that the positivity conditions of boundary points in the ORCM are satisfied by calculated results on the positivity conditions, while the positivity conditions of boundary points in the CM are not satisfied. The boundary value problems on the 2-D and 3-D Poisson's equations and the 3-D Helmholtz's equation are analyzed by using the ORCM and the CM. The numerical solutions by using both the ORCM and the CM are compared with the exact solutions. The relative errors by using the ORCM are smaller than those by using the CM, for both the unknown variables and their derivatives of 2-D problems and for the unknown variables of 3-D problems, and the relative errors of the unknown's derivatives of 3-D problems by using the ORCM are about same as those by using the CM. Convergence studies in the numerical examples show that the ORCM possesses good convergence for both the unknown variables and their derivatives. Because the ORCM does not demand any specific type of partial differential equations, it is concluded that the ORCM promises the wide engineering applications.
\end{abstract}

Keywords : Meshless method, Collocation method, Positivity conditions, Over-range points, Kronecker-delta

\section{Introduction}

A lot of meshless methods or meshfree methods have been proposed. The early representatives of these methods are the diffuse element method (Nayroles, et al., 1992), the element free Galerkin method (Belytschko, et al., 1994), the reproducing kernel particle method (Liu, et al., 1995), the finite point method (Oñate, et al., 1996a), the hp-clouds method (Duarte and Oden, 1996), the partition of unity method (Melenk and Babuska, 1996), the meshless local Petrov-Galerkin (MLPG) approach (Atluri and Zhu, 1998), the local boundary integral equation method (Zhu, et al., 1998), and the point collocation method (PCM) based on reproducing kernel approximations (Aluru, 2000). While, in the meshless techniques, complicated non-polynomial interpolation functions are used which render the integration of the weak form rather difficulty. Failure to perform the integration accurately results in loss of accuracy. The integration of complicated non-polynomial interpolation function also costs much CPU time. The collocation method has no issues of the integration scheme and so on. Many collocation methods based on different types of approximations or interpolations have been proposed in the literature. Oñate et al. (Oñate, et al., 1996a) have proposed a finite point method based on weighted least squares interpolations. Oñate et al. (Oñate, et al., 1996b) have also proposed a residual stabilization procedure, adequate for the finite point method, and further extended the finite point method to the solution of the advective-convective transport equations as well as those governing the flow of compressible fluids. 
Aluru (Aluru, 2000) has presented a point collocation method based on reproducing kernel approximations. Jin et al. (Jin, et al., 2004) have shown the robustness of collocation meshless methods can be improved by ensuring that the positivity conditions are satisfied when constructing approximation functions and their derivatives. Boroomand et al. (Boroomand, et al., 2005) have presented a stabilized version of the finite point method to eliminate the ill-conditioning effect. Atluri et al. (Atluri, et al., 2006a) have presented a MLPG mixed collocation method by using the Dirac delta function as the test function in the MLPG method, and shown that the MLPG mixed collocation method is more efficient than the other MLPG implementations. Atluri et al. (Atluri, et al., 2006b) have proposed a finite difference method, within the framework of the MLPG approach. The MLPG mixed collocation method has also been successfully used in material orientation and topology optimization (Li and Atluri, 2008). Chen et al. (Chen, et al., 2009) have proposed subdomain radial basis collocation method, in which the approximation in each subdomain is constructed separately. $\mathrm{Hu}$ et al. (Hu, et al., 2011) have performed the perturbation and stability analysis of the reproducing kernel collocation method. $\mathrm{Xu}$ et al. (Xu, et al., 2012) have developed virtual boundary meshless least square collocation method for calculation of two-dimensional multi-domain elastic problems. Chi et al. (Chi, et al., 2013) have proposed gradient reproducing kernel collocation method which can reduces the order of differentiation to the first order for solving second-order partial differential equations with strong form collocation.

To improve the robustness of the collocation methods (CM), Jin et al. (Jin, et al., 2004) have proposed techniques, based on modification of weighting functions, to ensure satisfaction of positivity conditions when using a scattered set of points. The positivity conditions are some inequalities on the shape function and its second-order derivatives. Patankar (Patankar, 1980) included the positivity conditions in a series of basic rules for the construction of finite differences and pointed out that the consequence of violating the positivity conditions give a physically unrealistic solution. It has been shown that the satisfaction of the positivity conditions ensures the convergence of the finite difference method with arbitrary irregular meshes for some class of elliptic problems (Demkowicz, et al., 1984). It has been shown that the significance of the positivity conditions in meshless collocation approaches, and violation of the positivity conditions can significantly result in a large error in the numerical solution (Jin, et al., 2004). For boundary points, however, the positivity conditions cannot be satisfied, obviously. To overcome the demerit of CM, the over-range collocation method (ORCM) has been proposed (Guo, 2011). In ORCM, some over-range collocation points are introduced which are located at outside of domain of the analyzed body, and at the over-range collocation points no satisfaction of any governing partial differential equation or boundary condition is needed. While some boundary value problems on nonlinear partial differential equations (Guo, et al., 2013) have been analyzed by using the ORCM, and it has been shown that the ORCM works well for those boundary value problems, the accuracy comparison of the ORCM and the $\mathrm{CM}$ is an open question yet. In this paper, it is shown that the positivity conditions of boundary points in the ORCM are satisfied by calculated results on the positivity conditions, but the positivity conditions of boundary points in the CM are not satisfied. The boundary value problems on Poisson's equations of 2-D and 3-D and Helmholtz's equation of 3-D are analyzed by using the ORCM and the CM. The numerical solutions by using both the ORCM and the CM are compared with the exact solutions.

\section{Formulation of the ORCM}

\subsection{Collocation Scheme}

Let us assume a scalar problem governed by a partial differential equation:

$$
D(u)=b, \quad \text { over } \Omega
$$

with boundary conditions

$$
\begin{aligned}
& T(u)=t, \quad \text { on } \Gamma_{t} \\
& u-u_{c}=0, \quad \text { on } \Gamma_{u}
\end{aligned}
$$

to be satisfied in a domain $\Omega$ with boundary $\Gamma=\Gamma_{t} \cup \Gamma_{u}$, where $D$ and $T$ are appropriate differential operators, $u$ is the problem unknown function, $b$ and $t$ are external forces or sources acting over $\Omega$ and along $\Gamma_{t}$, respectively. $u_{c}$ is the assigned value of $u$ over $\Gamma_{u}$.

Consider taking some collocation points in $\Omega$, at which Eq. (1) is satisfied, and some collocation points on $\Gamma_{t}$, at which both Eq. (1) and Eq. (2) are satisfied, as well as some collocation points on $\Gamma_{u}$, at which both Eq. (1) and Eq. (3) 
are satisfied. Besides the collocation points over $\Omega$, let us assume other points located at outside of $\Omega$ and call them over-range points. At outside of $\Omega$, neither satisfaction of any governing partial differential equation nor satisfaction of boundary condition is needed. Because the over-range points are used in interpolating calculation of boundary points, the unsatisfactory issue of the positivity conditions of boundary points in collocation methods can be avoided by using the over-range points.

Let us assume that the number of points in domain is $K_{d}$, the number of boundary points is $K_{b}$ and the number of over-range points is $K_{o}$, then the number of unknown variables is $K_{d}+K_{b}+K_{o}$ for a scalar problem. Because the number of equations of the ORCM is $K_{d}+2 K_{b}$ for the scalar problem, by taking the same number of the equations with that of the unknown variables, we obtain that the number of the over-range points $K_{o}$ must be equal to the number of boundary points $K_{b}$.

\subsection{The MLS approximation with Kronecker-Delta property}

In the classical moving least-square (MLS) approximation, the shape functions have no Kronecker-delta property, so that the essential node condition cannot be imposed on boundaries. In this paper, a modified MLS approximation is used, where its shape functions have Kronecker-delta property. Therefore, the unsatisfactory issue of the essential node condition can be avoided in the modified MLS approximation.

Consider a small domain $\Omega_{x}$, the neighborhood of a point $x_{1}$, which is located in $\Omega$ or on $\Gamma$ (the over-range points never become point $x_{1}$ ). Over a number of randomly located nodes $\left\{x_{i}\right\}, i=1,2, \cdots, n$, the MLS approximation $u^{h}$ of $u$ can be defined by

$$
u^{h}(\boldsymbol{x})=\boldsymbol{p}^{T}(\boldsymbol{x}) \boldsymbol{\alpha}, \quad \forall \boldsymbol{x} \in \Omega_{x}
$$

where $\boldsymbol{p}^{T}(\boldsymbol{x})=\left[\begin{array}{llll}p_{1}(\boldsymbol{x}) & p_{2}(\boldsymbol{x}) & \cdots & p_{m}(\boldsymbol{x})\end{array}\right]$ is a complete monomial basis of order $m$ which is a function of the space coordinates $\boldsymbol{x}=\left[\begin{array}{lll}x & y & z\end{array}\right]^{T} . \boldsymbol{\alpha}$ is a vector of unknown polynomial coefficients.

$$
\boldsymbol{\alpha}=\left[\begin{array}{llll}
\alpha_{1} & \alpha_{2} & \cdots & \alpha_{m}
\end{array}\right]^{T}
$$

For example, for a 3-D problem,

$$
\boldsymbol{P}^{T}(\boldsymbol{x})=\left[\begin{array}{llllllllll}
1 & x & y & z & x^{2} & y^{2} & z^{2} & x y & y z & z x
\end{array}\right]
$$

this is a quadratic basis, and $m=10$.

A weighted least-square solution is obtained for $\boldsymbol{\alpha}$ from the following system of $n$ equations in $m$ unknown ( $n$ is larger than $m)$ :

$$
\boldsymbol{u}^{h}=\boldsymbol{H} \boldsymbol{\alpha}
$$

where

$$
\boldsymbol{u}^{h}=\left[\begin{array}{llll}
u_{1}^{h} & u_{2}^{h} & \cdots & u_{n}^{h}
\end{array}\right]^{T}
$$

is a vector of the nodal MLS approximation of function $u$, and

$$
\boldsymbol{H}=\left[\begin{array}{c}
\boldsymbol{p}^{T}\left(x_{1}\right) \\
\boldsymbol{p}^{T}\left(\boldsymbol{x}_{2}\right) \\
\vdots \\
\boldsymbol{p}^{T}\left(\boldsymbol{x}_{n}\right)
\end{array}\right]_{n \times m}
$$

The classical least-square solution of the above over-constrained system does not guarantee exact satisfaction of any of the equations of Eq. (7). Non-satisfaction of the first equation would then mean $u_{1}^{h} \neq p^{T}\left(x_{1}\right) \alpha$. Hence, a different approach (Rajendran and Zhang, 2007) to weighted least-squares solution can be adopted: Out of the $n$ equations of Eq. 
(7), let the first equation (corresponding to node 1) be satisfied exactly and the rest in the least-square sense. This is done by using the first equation to eliminate $\alpha_{1}$ from the rest of equations:

$$
\alpha_{1}=u_{1}^{h}-\left(\alpha_{2} x_{1}+\alpha_{3} y_{1}+\alpha_{4} z_{1}+\alpha_{5} x_{1}^{2}+\alpha_{6} y_{1}^{2}+\alpha_{7} z_{1}^{2}+\alpha_{8} x_{1} y_{1}+\alpha_{9} y_{1} z_{1}+\alpha_{10} z_{1} x_{1}\right)
$$

Substituting for $\alpha_{1}$ in Eq. (7), the reduced system of equations can be obtained:

$$
\overline{\boldsymbol{u}}^{h}=\overline{\boldsymbol{H}} \overline{\boldsymbol{\alpha}}
$$

where

$$
\begin{aligned}
& \overline{\boldsymbol{u}}^{h}=\left[\begin{array}{llll}
u_{2}^{h}-u_{1}^{h} & u_{3}^{h}-u_{1}^{h} & \cdots & u_{n}^{h}-u_{1}^{h}
\end{array}\right]^{T}
\end{aligned}
$$

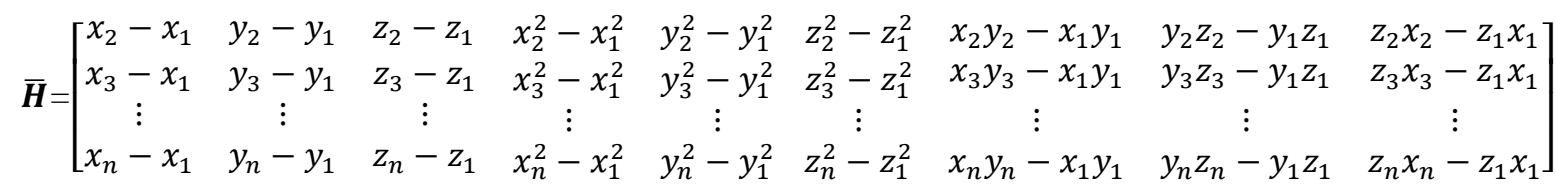

$$
\begin{aligned}
& \equiv\left[\begin{array}{c}
\overline{\boldsymbol{P}}^{T}\left(\boldsymbol{x}_{2}\right) \\
\overline{\boldsymbol{P}}^{T}\left(\boldsymbol{x}_{3}\right) \\
\vdots \\
\overline{\boldsymbol{P}}^{T}\left(\boldsymbol{x}_{n}\right)
\end{array}\right]_{(n-1) \times(m-1)} \\
& \overline{\boldsymbol{\alpha}}=\left[\begin{array}{llll}
\alpha_{2} & \alpha_{3} & \cdots & \alpha_{m}
\end{array}\right]^{T}
\end{aligned}
$$

The coefficient vector $\overline{\boldsymbol{\alpha}}$ is determined by minimizing a weighted discrete $L_{2}$ norm, defined as:

$$
J=\sum_{i=2}^{n} w\left(\boldsymbol{x}_{i}\right)\left[\overline{\boldsymbol{p}}^{T}\left(\boldsymbol{x}_{i}\right) \bar{\alpha}-\bar{u}_{i}\right]^{2}=[\overline{\boldsymbol{H}} \overline{\boldsymbol{\alpha}}-\overline{\boldsymbol{u}}]^{T} \boldsymbol{W}[\overline{\boldsymbol{H}} \overline{\boldsymbol{\alpha}}-\overline{\boldsymbol{u}}]
$$

where $w(\boldsymbol{x})$ is the weight function, with $w(\boldsymbol{x})>0$ for all nodes in the support of $w(\boldsymbol{x})$ (the support is considered to be equal to $\Omega_{x}$ in this paper), $\boldsymbol{x}_{i}$ denotes the value of $\boldsymbol{x}$ at node $i$, and the matrices $\boldsymbol{W}$ is defined as

$$
\begin{aligned}
& \boldsymbol{W}=\left[\begin{array}{cccc}
w\left(\boldsymbol{x}_{2}\right) & 0 & \cdots & 0 \\
0 & w\left(\boldsymbol{x}_{3}\right) & \cdots & 0 \\
\cdots & \cdots & \cdots & \cdots \\
0 & 0 & \cdots & w\left(\boldsymbol{x}_{n}\right)
\end{array}\right]_{(n-1) \times(n-1)} \\
& \bar{u}_{i}=\hat{u}_{i}-\hat{u}_{1}, \quad i=2,3, \cdots, n \\
& \overline{\boldsymbol{u}}=\left[\begin{array}{llll}
\hat{u}_{2}-\hat{u}_{1} & \hat{u}_{3}-\hat{u}_{1} & \cdots & \hat{u}_{n}-\hat{u}_{1}
\end{array}\right]^{T}
\end{aligned}
$$

where $\hat{u}_{i}, i=1,2, \cdots, n$, are the fictitious nodal values of the function $u$.

Minimizing $J$ in Eq. (15) with respect to $\overline{\boldsymbol{\alpha}}$ yields

$$
\begin{aligned}
& \overline{\boldsymbol{\alpha}}=\boldsymbol{A}^{-1} \boldsymbol{B} \overline{\boldsymbol{u}} \\
& \boldsymbol{B}=\overline{\boldsymbol{H}}^{T} \boldsymbol{W} \\
& \boldsymbol{A}=\boldsymbol{B} \overline{\boldsymbol{H}}
\end{aligned}
$$

Substituting Eq. (19) into Eq. (11) gives a relation which may be written as the form of an interpolation function, as

$$
\overline{\boldsymbol{u}}^{h}=\overline{\boldsymbol{H}} \boldsymbol{A}^{-1} \boldsymbol{B} \overline{\boldsymbol{u}}
$$

Equation (10) can be rewritten as: 


$$
\begin{aligned}
& \alpha_{1}=u_{1}^{h}-\boldsymbol{s}\left(\boldsymbol{x}_{1}\right) \bar{\alpha} \\
& \boldsymbol{s}\left(\boldsymbol{x}_{1}\right)=\left[\begin{array}{lllllllll}
x_{1} & y_{1} & z_{1} & x_{1}^{2} & y_{1}^{2} & z_{1}^{2} & x_{1} y_{1} & y_{1} z_{1} & z_{1} x_{1}
\end{array}\right]
\end{aligned}
$$

Equation (4) can be written as:

$$
\begin{aligned}
& u^{h}=\alpha_{1}+\boldsymbol{s}(\boldsymbol{x}) \bar{\alpha} \\
& \boldsymbol{s}(\boldsymbol{x})=\left[\begin{array}{llllllllll}
x & y & z & x^{2} & y^{2} & z^{2} & x y & y z & z x
\end{array}\right]
\end{aligned}
$$

Substituting Eq. (19) and Eq. (23) into Eq. (25), the following equation can be obtained:

$$
\begin{aligned}
& u^{h}=u_{1}^{h}+\boldsymbol{q}(\boldsymbol{x}) \boldsymbol{A}^{-1} \boldsymbol{B} \overline{\boldsymbol{u}} \\
& \boldsymbol{q}(\boldsymbol{x})=\boldsymbol{s}(\boldsymbol{x})-\boldsymbol{s}\left(\boldsymbol{x}_{1}\right)
\end{aligned}
$$

Because

$$
\begin{aligned}
& \boldsymbol{q}\left(\boldsymbol{x}_{1}\right)=0 \\
& u^{h}\left(\boldsymbol{x}_{1}\right)=u_{1}^{h}
\end{aligned}
$$

$\widehat{\boldsymbol{u}}$ may be defined as

$$
\widehat{\boldsymbol{u}}=\left[\begin{array}{llll}
\hat{u}_{1} & \hat{u}_{2} & \cdots & \hat{u}_{n}
\end{array}\right]^{T}
$$

then, from Eq. (27), the following equation may be obtained:

$$
\begin{aligned}
& u^{h}=\boldsymbol{N}(\boldsymbol{x}) \widehat{\boldsymbol{u}} \\
& \boldsymbol{N}(\boldsymbol{x})=\left[1-\left(\underset{1 \times(\mathrm{m}-1)}{\mathbf{q}(\mathbf{x})} \underset{(\mathrm{m}-1) \times(m-1)}{\underset{\mathbf{A}^{-1}}{\mathbf{B}}} \underset{(\mathrm{m}-1) \times(n-1)}{\mathbf{B}} \underset{(\mathrm{n}-1) \times 1}{\mathbf{1}}\right) \underset{1 \times(\mathrm{m}-1)}{\mathbf{q}(\mathbf{x})} \underset{(\mathrm{m}-1) \times(m-1)}{\mathbf{A}^{-1}} \underset{(\mathrm{m}-1) \times(n-1)}{\mathbf{B}}\right]
\end{aligned}
$$

In Eq. (33), 1 is vector of dimension ( $n-1)$ with all entries being equal to unity.

Recall from Eq. (29), using this result in Eq. (33), the Kronecker-delta property of $\boldsymbol{N}(\boldsymbol{x})$ may be established:

$$
\boldsymbol{N}\left(\boldsymbol{x}_{1}\right)=\left[\begin{array}{lllll}
1 & 0 & 0 & \cdots & 0
\end{array}\right]
$$

It means that at node 1 , the shape function for node 1 takes a value of unity and all other shape function take zero values. Therefore, Eq. (33) is the shape functions of the MLS approximation with Kronecker-delta property.

From Eq. (32) and Eq. (30), the following result can be obtained:

$$
\hat{u}_{1}=u^{h}\left(\boldsymbol{x}_{1}\right)=u_{1}^{h}
$$

In this paper, the weight functions $w(\boldsymbol{x})$ may use a spline function as follows:

$$
\begin{aligned}
& w(\boldsymbol{x})=1-6\left(\frac{d}{r}\right)^{2}+8\left(\frac{d}{r}\right)^{3}-3\left(\frac{d}{r}\right)^{4}, \quad 0 \leq d \leq r \\
& w(\boldsymbol{x})=0, \quad d \geq r
\end{aligned}
$$

where $d=\left|\boldsymbol{x}-\boldsymbol{x}_{1}\right|$ is the distance from point $x$ to the center node $x_{1}$, and $r$ is the radius of $\Omega_{x}$, which is taken as a circle for 2-D problems and a sphere for 3-D problems, respectively, and its center is the point $x_{1}$. 
As the MLS approximation $u^{h}$ of $u$, Eq. (32) can be substituted into Eqs. (1), (2) and (3). Let the residuals from the substituting be equal to zero at $n_{t}\left(n_{t}=K_{d}+K_{b}\right)$ collocation points that are located at $\Omega$ or $\Gamma$, never at outside of $\Omega$, therefore, by using Eq. (32) the algebraic equations on $\hat{u}_{i},\left(i=1,2, \cdots, n_{f}, n_{f}=n_{t}+K_{o}\right.$ is the total number of nodes), in which $\hat{u}_{i}$ consists of $\hat{u}_{i}^{(\Omega)}\left(i=1,2, \cdots, n_{t}\right)$ and $\hat{u}_{i}^{(\bar{\Omega})}\left(i=n_{t}+1, n_{t}+2, \cdots, n_{f}\right)$ (where $\hat{u}_{i}^{(\Omega)}$ denotes $\hat{u}_{i}$ of the nodes over $\Omega$, and $\hat{u}_{i}^{(\bar{\Omega})}$ denotes $\hat{u}_{i}$ of the over-range nodes), can be obtained as follows:

$$
\begin{aligned}
& (D(\boldsymbol{N} \widehat{\boldsymbol{u}})-b)_{j}=0, \quad j=1,2, \cdots, n_{t} \\
& (T(\boldsymbol{N} \widehat{\boldsymbol{u}})-t)_{k}=0, \quad k=1,2, \cdots, K_{b 1} \\
& \left(\boldsymbol{N} \widehat{\boldsymbol{u}}-u_{c}\right)_{l}=0, \quad l=1,2, \cdots, K_{b 2}
\end{aligned}
$$

where $K_{b 1}+K_{b 2}=K_{b}$. The total number of Eqs. (37), (38) and (39) is $n_{e}=n_{t}+K_{b}$, but let us take $n_{e}=n_{f}$. It can be seen that there is no any the residual equation of the over-range node in Eqs. (37), (38) and (39), and $\hat{u}_{i}^{(\Omega)}$ in these equations are only used in interpolating calculation of $u^{h}$ in those $\Omega_{x}$ of nodes $x_{1}$ on $\Gamma$ (see the red nodes in Figs. 3 and 4) by Eq. (32).

To understand how the over-range nodes are used in the discrete equations, we can consider roles of Gauss points and nodes in weak form methods such as FEM. Gauss points are used in physics sense, and the nodes in the analyzed domain are only used in interpolating calculation in FEM, and it is known that no over-constrained condition in physics sense is imposed into FEM by these nodes. Then, we can say that the role of the over-range nodes in the ORCM resembles that of the nodes in the domain in FEM to some extent. Therefore, it can be understood that the over-range nodes are not used in physics sense, no over-constrained condition in physics sense is imposed into the boundary value problem.

\subsection{The local coordinate system}

As anisotropy of the point distribution in $\Omega_{x}$, matrix $\boldsymbol{A}$ in Eq. (21) becomes ill-conditioned and the quality of the approximation deteriorates. In order to prevent such undesirable effect, a local coordinate system $\xi, \eta$, $\zeta$ is chosen with origin at the node $x_{1}$,

$$
\begin{gathered}
\xi=\frac{x-x_{1}}{R_{x}} \\
\eta=\frac{y-y_{1}}{R_{y}} \\
\zeta=\frac{z-z_{1}}{R_{z}}
\end{gathered}
$$

where $R_{x}, R_{y}$ and $R_{z}$ denote maximum distances along $x, y$ and $z$ measured from the point $\boldsymbol{x}_{1}$ to exterior nodes in $\Omega_{x}$. In Eq. (36a), the spline function has now the following form in terms of the local coordinates:

$$
w(\xi)=1-6\left(\frac{\xi^{2}+\eta^{2}+\zeta^{2}}{\rho^{2}}\right)+8\left(\frac{\xi^{2}+\eta^{2}+\zeta^{2}}{\rho^{2}}\right)^{\frac{3}{2}}-3\left(\frac{\xi^{2}+\eta^{2}+\zeta^{2}}{\rho^{2}}\right)^{2}
$$

where $-1 \leq \xi \leq 1,-1 \leq \eta \leq 1,-1 \leq \zeta \leq 1$ as usual, $\rho$ is a constant that may control weight values of different nodes in $\Omega_{x} . \rho=6$ for 2-D problems and $\rho=10$ for 3-D problems are used in this paper. The matrix $\boldsymbol{A}$ is not dependent on the dimensions of $\Omega_{x}$ any longer. The approximate function is also expressed in terms of the local coordinate as

$$
u^{h}(\xi)=N(\xi) \widehat{\boldsymbol{u}}
$$

$\boldsymbol{A}^{-1} \boldsymbol{B}$ in Eq. (33) can be defined as $\boldsymbol{C}$ : 


$$
\boldsymbol{C}=\boldsymbol{A}^{-1} \boldsymbol{B}
$$

Then, from Eq. (33), entries of $\boldsymbol{N}(\boldsymbol{x})$ for the quadratic basis $(m=10)$ can be written as:

$$
\begin{aligned}
N_{1}(\boldsymbol{x})= & -\left[\left(x-x_{1}\right) \sum_{i=1}^{n-1} C_{1 i}+\left(y-y_{1}\right) \sum_{i=1}^{n-1} C_{2 i}+\left(z-z_{1}\right) \sum_{i=1}^{n-1} C_{3 i}\right. \\
& +\left(x^{2}-x_{1}^{2}\right) \sum_{i=1}^{n-1} C_{4 i}+\left(y^{2}-y_{1}^{2}\right) \sum_{i=1}^{n-1} C_{5 i}+\left(z^{2}-z_{1}^{2}\right) \sum_{i=1}^{n-1} C_{6 i} \\
& \left.+\left(x y-x_{1} y_{1}\right) \sum_{i=1}^{n-1} C_{7 i}+\left(y z-y_{1} z_{1}\right) \sum_{i=1}^{n-1} C_{8 i}+\left(z x-z_{1} x_{1}\right) \sum_{i=1}^{n-1} C_{9 i}\right] \\
N_{i+1}(\boldsymbol{x})= & \left(x-x_{1}\right) C_{1 i}+\left(y-y_{1}\right) C_{2 i}+\left(z-z_{1}\right) C_{3 i}+\left(x^{2}-x_{1}^{2}\right) C_{4 i} \\
& +\left(y^{2}-y_{1}^{2}\right) C_{5 i}+\left(z^{2}-z_{1}^{2}\right) C_{6 i}+\left(x y-x_{1} y_{1}\right) C_{7 i} \\
& +\left(y z-y_{1} z_{1}\right) C_{8 i}+\left(z x-z_{1} x_{1}\right) C_{9 i} \quad(i=1,2, \cdots, n-1)
\end{aligned}
$$

where $C_{j i},(j=1,2, \cdots, 9 ; i=1,2, \cdots, n-1)$ are entries of $\boldsymbol{C}$.

At the point $x_{1}$, because $\xi_{1}=0, \eta_{1}=0, \zeta_{1}=0$ then the first-order derivatives of the shape function with the local coordinates can be obtained from Eqs. (44) and (45):

$$
\begin{aligned}
& \frac{\partial N\left(\xi_{1}\right)}{\partial \xi}=\left[\begin{array}{lllll}
-\sum_{i=1}^{n-1} C_{1 i} & C_{11} & C_{12} & \cdots & C_{1(n-1)}
\end{array}\right] \\
& \frac{\partial N\left(\xi_{1}\right)}{\partial \eta}=\left[\begin{array}{lllll}
-\sum_{i=1}^{n-1} C_{2 i} & C_{21} & C_{22} & \cdots & C_{2(n-1)}
\end{array}\right] \\
& \frac{\partial N\left(\xi_{1}\right)}{\partial \zeta}=\left[\begin{array}{lllll}
-\sum_{i=1}^{n-1} C_{3 i} & C_{31} & C_{32} & \cdots & C_{3(n-1)}
\end{array}\right]
\end{aligned}
$$

From Eqs. (46), (47) and (48), we may see that formulas of the shape function derivatives with the local coordinates are very simple, and in fact, it is a merit of the ORCM using the local coordinates.

\subsection{The positivity conditions}

The positivity conditions (Jin, et al., 2004) on the approximation function $N_{i}$ (x) of Eq. (33) and its second-order derivatives are stated as,

$$
\begin{aligned}
& N_{i}\left(\boldsymbol{x}_{j}\right) \geq 0 \\
& \nabla^{2} N_{i}\left(\boldsymbol{x}_{j}\right) \geq 0, j \neq i \\
& \nabla^{2} N_{i}\left(\boldsymbol{x}_{i}\right)<0
\end{aligned}
$$

where $N_{i}\left(\boldsymbol{x}_{j}\right)$ is the approximation function of a point $i$ evaluated at a point $j$.

In the $\mathrm{CM}$, for a point $x_{1}$ on $\Gamma$, because no over-range point is used in its $\Omega_{x}$, the positivity conditions on the boundary point cannot be satisfied. To show the data, we have calculated the positivity conditions of 2-D problem in this case. In Figs. 1 and 2, nodes of $n=3 \times 3=9$ is used for each small domain $\Omega_{x}$ without over-range points, and the red point on $\Gamma$ is node $x_{1}$. The local coordinate system is used, then the value of shape function $N_{1}\left(\xi_{j}\right),(j=$ $1,2, \cdots, 9)$ and the values of $\nabla^{2} N_{1}\left(\xi_{j}\right),(j=1,2, \cdots, 9)$ are shown in Tables 1 and 2 (where subscript symbol 1 means node $x_{1}$ in $\Omega_{x}$ ). From Tables 1 and 2 , it is seen that the positivity conditions of these nodes are not satisfied. 


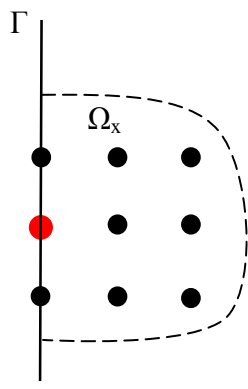

$\Omega$

Fig. $1 \Omega_{x}$ without over-range points of $x_{1}$ (the red node) on $\Gamma$ of line boundary for 2-D problem.

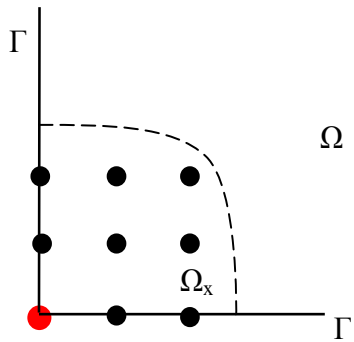

Fig. $2 \Omega_{x}$ without over-range points of $x_{1}$ (the red node) on $\Gamma$ of corner boundary for 2-D problem.

Table 1 Values of $N_{1}\left(\xi_{j}\right)$ and $\nabla^{2} N_{1}\left(\xi_{j}\right)$ without using over-range points in case of line boundary for 2-D problem.

\begin{tabular}{|c|c|c|c|c|c|c|c|c|c|}
\hline$j$ & 1 & 2 & 3 & 4 & 5 & 6 & 7 & 8 & 9 \\
\hline$N_{1}\left(\xi_{j}\right)$ & 1.0 & 0.0 & 0.0 & 0.0 & 0.0 & 0.0 & 0.0 & 0.0 & 0.0 \\
\hline$\nabla^{2} N_{1}\left(\xi_{j}\right)$ & 1.285 & 1.357 & -2.064 & 1.707 & -3.872 & 0.587 & 1.357 & -2.064 & 1.707 \\
\hline
\end{tabular}

Table 2 Values of $N_{1}\left(\xi_{j}\right)$ and $\nabla^{2} N_{1}\left(\xi_{j}\right)$ without using over-range points in case of corner boundary for 2-D problem.

\begin{tabular}{|c|c|c|c|c|c|c|c|c|c|}
\hline$j$ & 1 & 2 & 3 & 4 & 5 & 6 & 7 & 8 & 9 \\
\hline$N_{1}\left(\xi_{j}\right)$ & 1.0 & 0.0 & 0.0 & 0.0 & 0.0 & 0.0 & 0.0 & 0.0 & 0.0 \\
\hline$\nabla^{2} N_{1}\left(\xi_{j}\right)$ & 3.439 & -2.177 & 2.738 & -2.177 & -5.047 & -0.776 & 2.738 & -0.776 & 2.038 \\
\hline
\end{tabular}

To overcome the demerit of the classic collocation methods, besides the collocation points over $\Omega$, let us assume other collocation points located at outside of $\Omega$ and call them over-range points, at which no satisfaction of any governing partial differential equation or boundary condition is needed. By introducing some over-range points of $\Omega$ in the $\Omega_{x}$, the unsatisfactory issue of the positivity conditions of the boundary point can be avoided in the ORCM. To show the data, we have also calculated the positivity conditions of regular nodal model in this case. Figure 3 shows $\Omega_{x}$ with over-range points of $x_{1}$ (the red node) on $\Gamma$ and over-range nodes (the blue nodes) in $\Omega_{x}$. The value of shape function $N_{1}\left(\xi_{j}\right),(j=1,2, \cdots, 9)$ and the values of $\nabla^{2} N_{1}\left(\xi_{j}\right),(j=1,2, \cdots, 9)$ in this case are shown in Table 3 . From Table 3, it is seen that the positivity conditions of these nodes are satisfied. Because the over-range points are used only in interpolating calculation in $\Omega_{x}$ of $x_{1}$, and they never become any node $x_{1}$, the over-range points are not used in physics sense. 


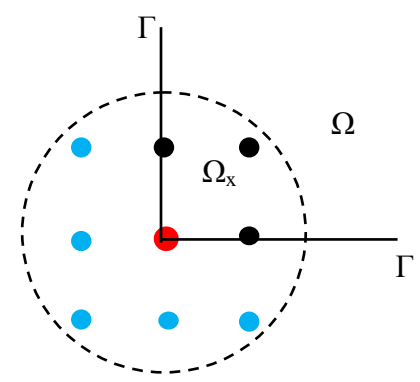

Fig. $3 \Omega_{x}$ with over-range points of $x_{1}$ (the red node) on $\Gamma$ and over-range nodes (the blue nodes) in $\Omega_{x}$ for 2-D problem.

Table 3 Values of $N_{1}\left(\xi_{j}\right)$ and $\nabla^{2} N_{1}\left(\xi_{j}\right)$ with using over-range points for 2-D problem.

\begin{tabular}{|c|c|c|c|c|c|c|c|c|c|}
\hline$j$ & 1 & 2 & 3 & 4 & 5 & 6 & 7 & 8 & 9 \\
\hline$N_{1}\left(\xi_{j}\right)$ & 1.0 & 0.0 & 0.0 & 0.0 & 0.0 & 0.0 & 0.0 & 0.0 & 0.0 \\
\hline$\nabla^{2} N_{1}\left(\xi_{j}\right)$ & -2.443 & 0.389 & 0.222 & 0.389 & 0.222 & 0.389 & 0.222 & 0.389 & 0.222 \\
\hline
\end{tabular}

If even the positivity conditions are satisfied, number and position of the over-range points in $\Omega_{x}$ can be chosen arbitrarily to some extent. It has been shown (Guo, 2011) that the ORCM possesses good accuracy even if using irregularly distributed nodal model. Then, the ORCM is able to use for the problem with arbitrary shape of analysis domain, generally. For the problems in which nodes are distributed in unstructured manner and the boundaries with complex shape are given, we can, as examples, choose the numbers and positions of the over-range points in the small domains as shown in Fig.4.
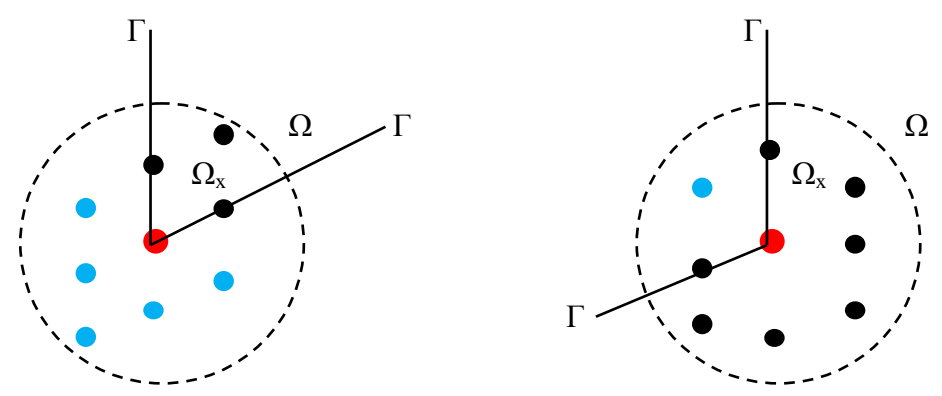

Fig. 4 Two examples of $\Omega_{x}$ with over-range points of $x_{1}$ (the red node) on $\Gamma$ with complex shape and over-range nodes (the blue points) in $\Omega_{x}$ for 2-D.

The satisfaction of the positivity conditions in Table 3 is shown only numerically for very simple cases, but because the local coordinate system is used, it is of certain generality. For the cases with complex boundary shape such as the cases shown in Fig. 4, some nodal distributions which are similar to the case shown in Fig. 3 can be easily arranged in small $\Omega_{x}$, like the nodal distributions shown in Fig. 4, so the positivity conditions for the cases may be satisfied. Although the positivity conditions are numerical conditions, and it is difficulty to show whether or not the positivity conditions for general cases are satisfied theoretically, it is easy to show numerically no satisfaction of the positivity conditions for general cases in the CM shown in Figs. 1 and 2. On the other hands, the positivity conditions are satisfied for all nodes over $\Omega$ by using the ORCM even for $\Omega$ with complex shape.

\section{Numerical Implementation}

\subsection{Poisson's Boundary Value Problems of 2-D}

For the purpose of error estimation and convergence studies, the Sobolev norm, $\|u\|_{0}$ of function $u$ and the norm of the first-order derivative vector of $u,\|\boldsymbol{q}\|_{0}$ are calculated. These norms are defined as 


$$
\begin{aligned}
& \|u\|_{0}=\left(\int_{\Omega} u^{2} d \Omega\right)^{1 / 2} \\
& \|\boldsymbol{q}\|_{0}=\left(\int_{\Omega} \boldsymbol{q}^{T} \cdot \boldsymbol{q} d \Omega\right)^{1 / 2} \\
& \boldsymbol{q}=\left[\begin{array}{ll}
\frac{\partial u}{\partial x} & \frac{\partial u}{\partial y}
\end{array}\right]^{T}=\left[\begin{array}{ll}
q_{x} & q_{y}
\end{array}\right]^{T}
\end{aligned}
$$

The relative errors for $\|u\|_{0}$ and $\|\boldsymbol{q}\|_{0}$ are defined as

$$
\begin{aligned}
& R_{0}=\frac{\left\|u^{n u m}-u^{\text {exa }}\right\|_{0}}{\left\|u^{e x a}\right\|_{0}} \\
& R_{q}=\frac{\left\|\boldsymbol{q}^{\text {num }}-\boldsymbol{q}^{\text {exa }}\right\|_{0}}{\left\|\boldsymbol{q}^{\text {exa }}\right\|_{0}}
\end{aligned}
$$

where $u^{\text {num }}$ and $\boldsymbol{q}^{\text {num }}$ are numerical solutions of $u$ and $\boldsymbol{q}$, respectively, and $u^{\text {exa }}$ and $\boldsymbol{q}^{\text {exa }}$ are the exact solutions of $u$ and $\boldsymbol{q}$, respectively.

A 2-D Poisson's equation

$$
\nabla^{2} u=-10\left(x^{2}+y^{2}\right)
$$

is analyzed over $(0,0) \times(1,1)$ domain by using the ORCM and the CM, and its numerical solutions are compared with an exact solution, which is chosen as follows:

$$
u=-x^{4}-y^{4}+x^{3} y+x^{2} y^{2}-x y^{3}
$$

Two mixed problems, the first mixed problem (according to Eq. (58), the essential boundary condition is imposed at nodes on left and right boundaries and the flux boundary condition $\left(q_{y}=t_{y}\right)$ is prescribed at nodes on top and bottom boundaries) and the second mixed problem (according to Eq. (58), the essential boundary condition is imposed at nodes on top and bottom boundaries and the flux boundary condition $\left(q_{x}=t_{x}\right)$ is prescribed at nodes on left and right boundaries), are solved, where $t_{x}$ and $t_{y}$ are the assigned values of $q_{x}$ and $q_{y}$ over $\Gamma_{t}$, respectively. For the ORCM, to meet the condition of $K_{o}=K_{b}, 8$ nodes are added to the boundary points, which are located on the boundary near the four corners of the domain, for all nodal models of the ORCM. Nodal models (including the over-range nodes $)$ of $129(11 \times 11+8)\left(K_{d}=7 \times 7, K_{b}=K_{o}=40\right)$ nodes, $177(13 \times 13+8)\left(K_{d}=9 \times 9, K_{b}=K_{o}=\right.$ 48) and $233(15 \times 15+8)\left(K_{d}=11 \times 11, K_{b}=K_{o}=56\right)$ nodes are used to study the convergence with nodal model refinement. Over-range points of one layer are used, and the over-range points are regularly located at outside of the domain. For the CM, in order to take the same nodal interval as the three nodal models of the ORCM, respectively, nodal models of $81(9 \times 9)$ nodes, $121(11 \times 11)$ nodes and $169(13 \times 13)$ nodes are used. Figure 5 shows nodal distribution of $233(15 \times 15+8)\left(K_{d}=11 \times 11, K_{b}=K_{o}=56\right)$ nodal model for the ORCM.

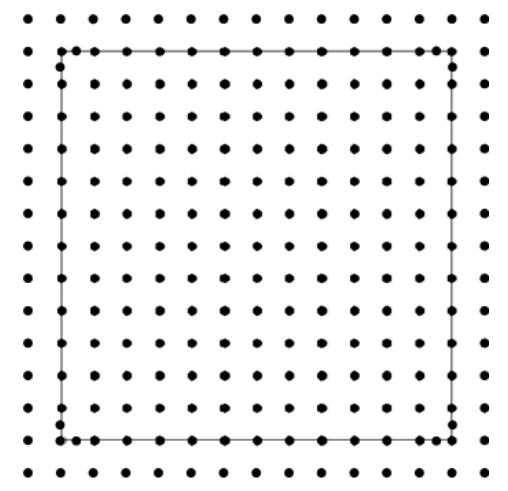

Fig. 5 Nodal distribution of $233(15 \times 15+8)\left(K_{d}=11 \times 11, K_{b}=K_{o}=56\right)$ node model for the ORCM. 
The results of relative errors of $R_{0}$ and $R_{q}$ by using the ORCM and the CM are shown in Fig. 6 and Fig. 7, respectively, for the first mixed problem (where $n_{a}$ is a total number of the nodes of 2-D problem except the over-range nodes). The results of relative errors of $R_{0}$ and $R_{q}$ by using the ORCM and the CM are shown in Fig. 8 and Fig. 9, respectively, for the second mixed problem. It is seen that for both mixed problems, the relative errors of both $R_{0}$ and $R_{q}$ by using the ORCM are smaller than those by using the CM, and the relative errors of both $R_{0}$ and $R_{q}$ become smaller with increase of $n_{a}$.

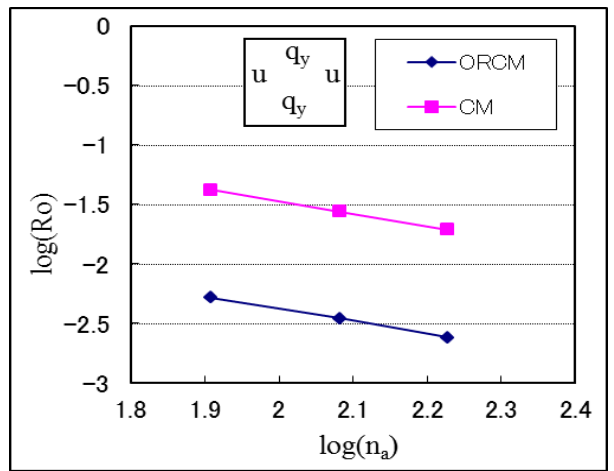

Fig. 6 Relative error $R_{0}$ and convergence for the first mixed problem of 2-D.

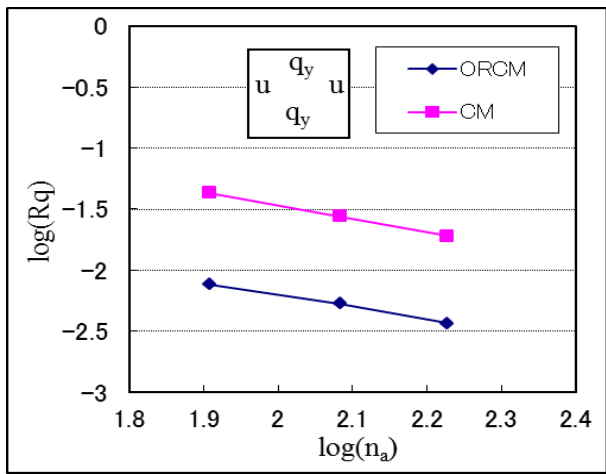

Fig. 7 Relative error $R_{q}$ and convergence for the first mixed problem of 2-D.

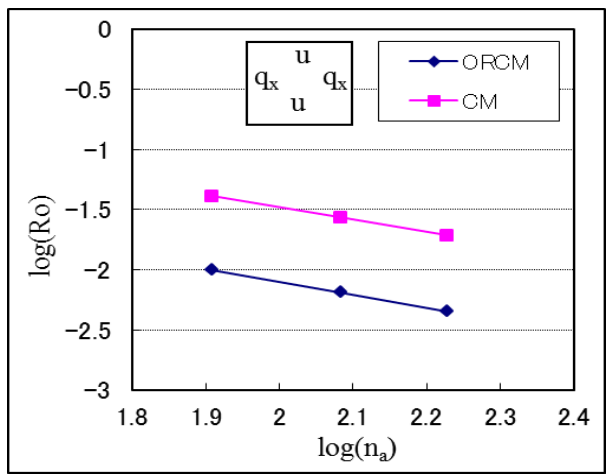

Fig. 8 Relative error $R_{0}$ and convergence for the second mixed problem of 2-D. 


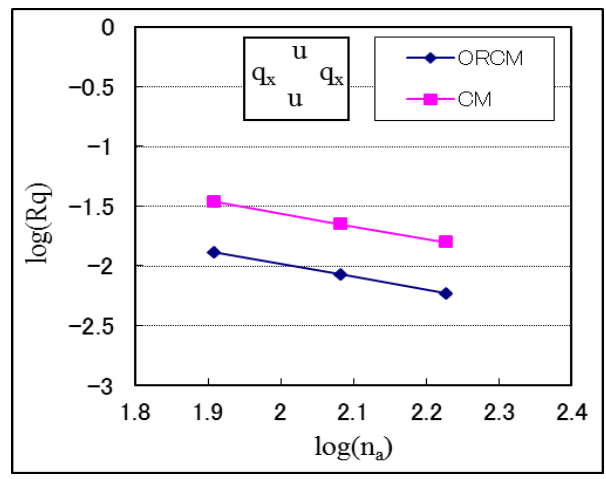

Fig. 9 Relative error $R_{q}$ and convergence for the second mixed problem of 2-D.

Figure 10 is a comparison figure of values of $u$ at $x=0.5$ by using the ORCM, the CM and the exact solution with nodal model of 177 nodes (121 nodes for the CM) for the first mixed problem. Figure 11 is a comparison figure of values of $\partial u / \partial y$ at $x=0.5$ by using the ORCM, the CM and the exact solution with nodal model of 177 nodes (121 nodes for the CM) for the second mixed problem. Figures 12(a), (b) and (c) are comparison figures of distributions of $u$ by using the ORCM, the CM and the exact solution with nodal model of 177 nodes (121 nodes for the CM) for the first mixed problem. It can be seen that the results of $u$ and $\partial u / \partial y$ by using the ORCM are more accurate than those by using the CM.

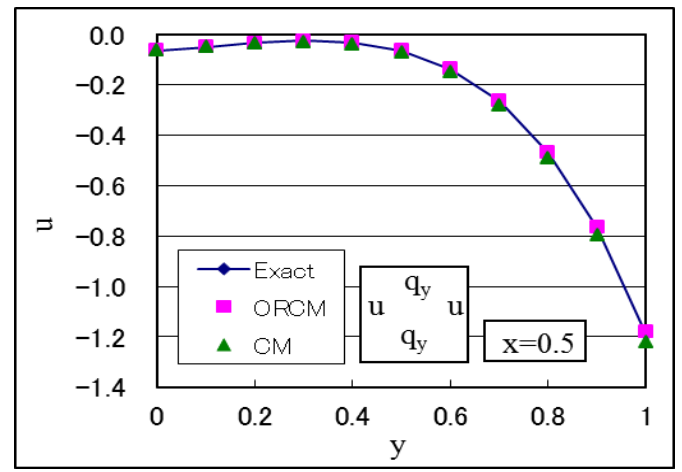

Fig. 10 Values of $u$ at $x=0.5$ by nodal model of 177 nodes ( 121 nodes for CM) for the first mixed problem.

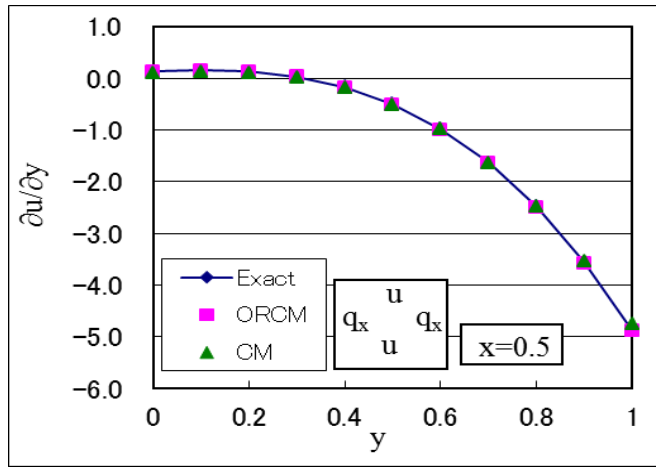

Fig. 11 Values of $\partial u / \partial y$ at $x=0.5$ by nodal model of 177 nodes ( 121 nodes for CM) for the second mixed problem. 


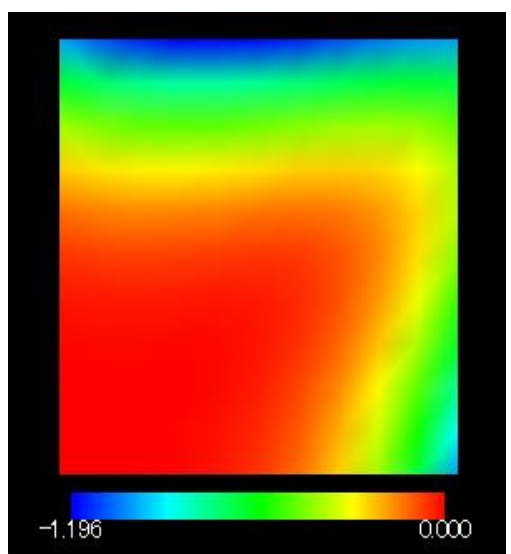

(a) ORCM

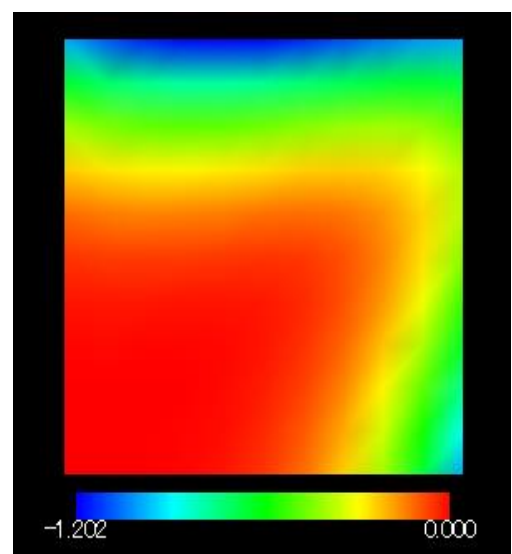

(b) Exact

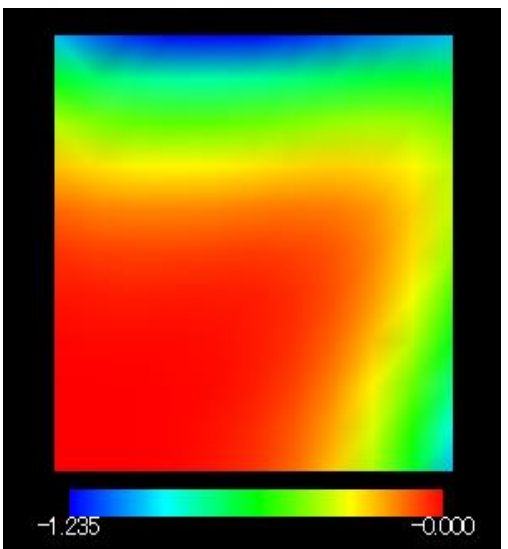

(c) $\mathrm{CM}$

Fig. 12 Distribution of values of $u$ by nodal model of 177 nodes (121 nodes for CM) for the first mixed problem.

\subsection{Poisson's and Helmholtz's Boundary Value Problems of 3-D}

For 3-D boundary value problems, $\|u\|_{0}$ and $\|\boldsymbol{q}\|_{0}$ are also calculated. These norms are defined as

$$
\begin{aligned}
& \|u\|_{0}=\left(\sum_{i=1}^{n_{t}} u_{i}^{2}\right)^{1 / 2} \\
& \|\boldsymbol{q}\|_{0}=\left(\sum_{i=1}^{n_{t}} \boldsymbol{q}_{i}^{T} \cdot \boldsymbol{q}_{i}\right)^{1 / 2} \\
& \boldsymbol{q}=\left[\begin{array}{lll}
\frac{\partial u}{\partial x} & \frac{\partial u}{\partial y} & \frac{\partial u}{\partial z}
\end{array}\right]^{T}=\left[\begin{array}{lll}
q_{x} & q_{y} & q_{z}
\end{array}\right]^{T}
\end{aligned}
$$

In order to avoid computing of 3-D numerical integration that costs very many CPU times, we use the norms of these non-integrate forms for error estimation of 3-D boundary value problems. The relative errors for $\|u\|_{0}$ and $\|\boldsymbol{q}\|_{0}$ are identically defined as Eqs. (55) and (56).

Analyzed domain is $(0.5,1.5) \times(0.5,1.5) \times(0.5,1.5)$ cube domain. For the ORCM, nodal models (including the over-range nodes $)$ of $223(5 \times 5 \times 5+98) \quad\left(K_{d}=3 \times 3 \times 3, K_{b}=K_{o}=98\right)$ nodes, $561(7 \times 7 \times 7+218)$ $\left(K_{d}=5 \times 5 \times 5, K_{b}=K_{o}=218\right)$ nodes and $1115(9 \times 9 \times 9+386)\left(K_{d}=7 \times 7 \times 7, K_{b}=K_{o}=386\right)$ nodes are used to study the convergence with nodal model refinement. Over-range points of one layer are used, and the over-range points are regularly located at outside of the domain. For the CM, in order to take the same nodal interval as the three nodal models of the ORCM, respectively, nodal models of $125(5 \times 5 \times 5)$ nodes, $343(7 \times 7 \times 7)$ nodes and $729(9 \times 9 \times 9)$ nodes are used.

\subsubsection{Poisson's Problem of 3-D}

A 3-D Poisson's equation

$$
\nabla^{2} u=\frac{1}{10}(x+y+z)
$$

is analyzed by using the ORCM and the CM, and its numerical solutions are compared with the exact solutions:

$$
u=-\frac{1}{12}\left(x^{3}+y^{3}+z^{3}\right)+\frac{3}{10}\left(x^{2} y+y^{2} z+z^{2} x\right)
$$

A mixed problem (the normal flux boundary condition is prescribed at nodes of $y=0.5$ and $y=1.5$ boundaries, the essential boundary condition is imposed at nodes of other boundaries) is solved. The results of relative errors of $R_{0}$ and $R_{q}$ by using the ORCM and the CM are shown in Fig. 13 and Fig. 14, respectively. It is seen that the relative errors of $R_{0}$ by using the ORCM are smaller than those by using the CM, the relative errors of $R_{q}$ by using the ORCM are about same as those by using the CM, and the relative errors of both $R_{0}$ and $R_{q}$ become smaller with 
increase of $n_{t}$.

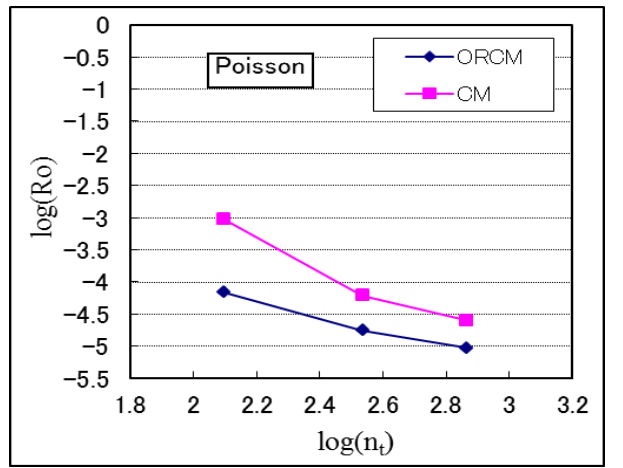

Fig. 13 Relative error $R_{0}$ and convergence for 3-D Poisson's problem.

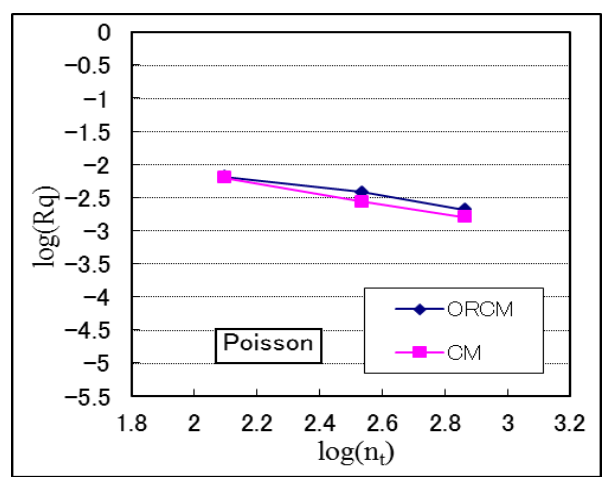

Fig. 14 Relative error $R_{q}$ and convergence for 3-D Poisson's problem.

Figure 15 is a comparison figure of values of $u$ at $x=1, z=1$ by using the ORCM, the CM and the exact solution with nodal model of 1115 nodes (729 nodes for the CM) for the first mixed problem. Figure 16 is a comparison figure of values of $\partial u / \partial y$ at $x=1, z=1$ by using the ORCM, the CM and the exact solution with nodal model of 1115 nodes (729 nodes for the CM) for the first mixed problem. Figures 17(a), (b) and (c) are comparison figures of distributions of $\partial u / \partial y$ at $x=1.5$ by using the ORCM, the CM and the exact solution with nodal model of 1115 nodes (729 nodes for the $\mathrm{CM}$ ) for the first mixed problem. It can be seen that some accurate results of $u$ and $\partial u / \partial y$ are obtained by using both the ORCM and the CM, but the minimum and maximum values of $\partial u / \partial y$ by using the ORCM are more accurate than those by using the CM as shown in Fig. 17.

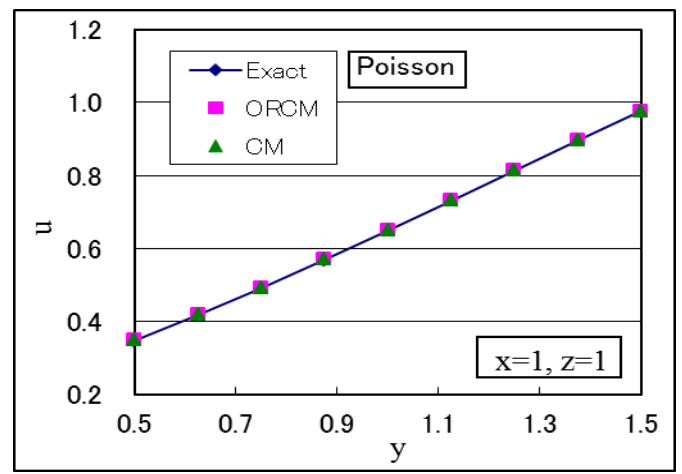

Fig. 15 Values of $u$ at $x=1, z=1$ by nodal model of 1115 nodes ( 729 nodes for CM) for the first mixed problem. 


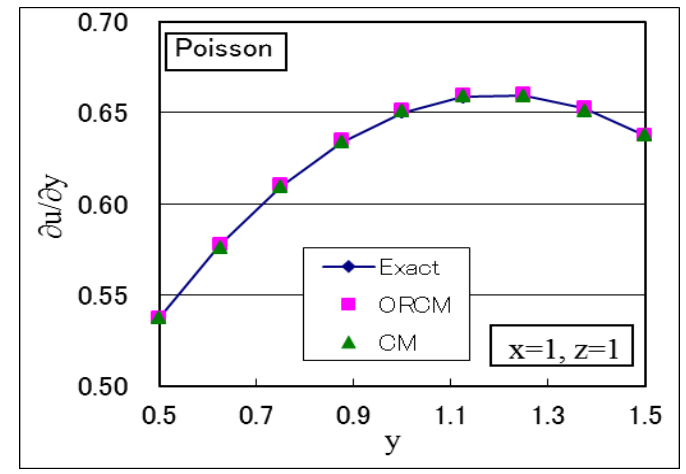

Fig. 16 Values of $\partial u / \partial y$ at $x=1, z=1$ by nodal model of 1115 nodes ( 729 nodes for $C M$ ) for the first mixed problem.

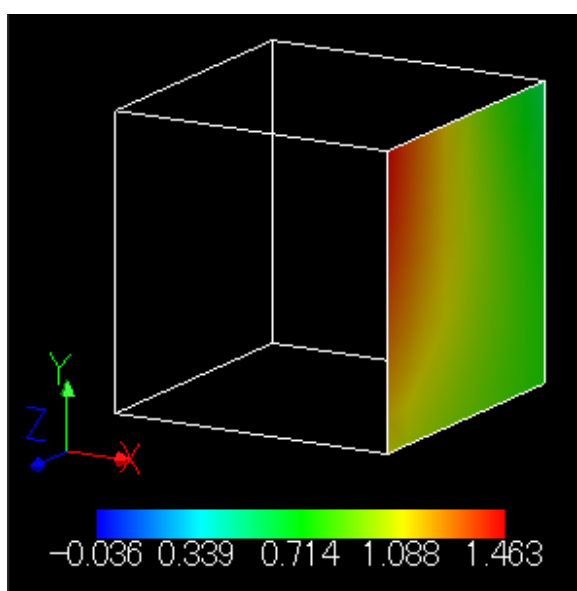

(a) ORCM

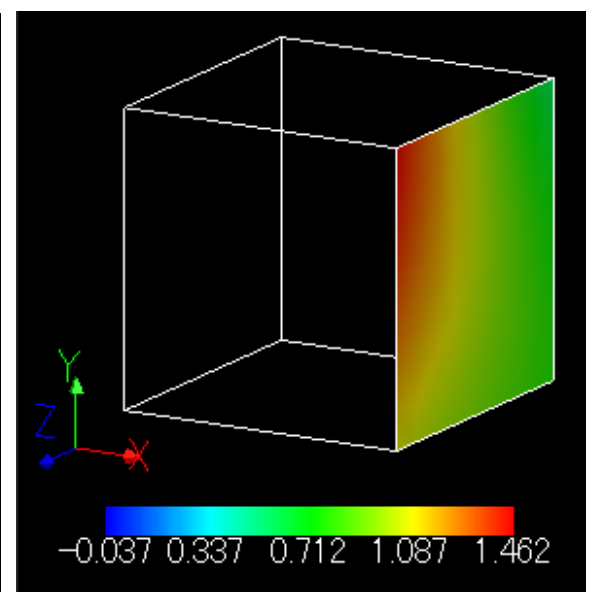

(b) Exact

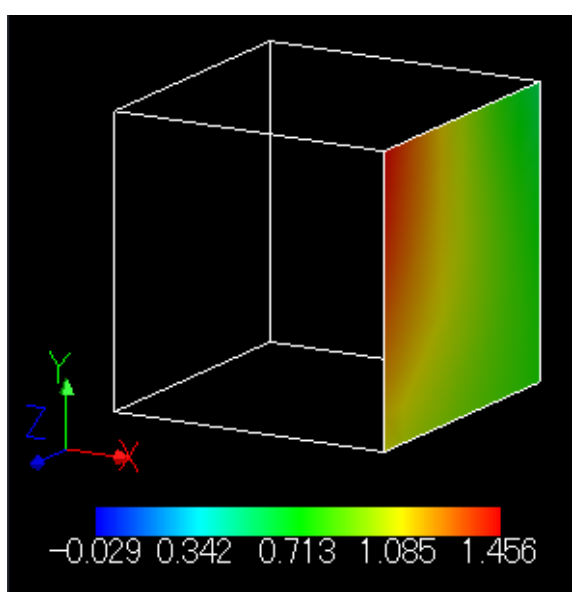

(c) $\mathrm{CM}$

Fig. 17 Values of $\partial \mathrm{u} / \partial \mathrm{y}$ at $x=1.5$ by nodal model of 1115 nodes ( 729 nodes for $\mathrm{CM}$ ) for the first mixed problem.

\subsubsection{Helmholtz's Problem of 3-D}

A 3-D Helmholtz's equation

$$
\nabla^{2} u+u=-x^{3}-y^{3}-z^{3}+3 x^{2} y+3 y^{2} z+3 z^{2} x
$$

is analyzed by using the ORCM and the CM, and its numerical solutions are compared with the exact solutions:

$$
u=-x^{3}-y^{3}-z^{3}+3 x^{2} y+3 y^{2} z+3 z^{2} x
$$


A mixed problem (the normal flux boundary condition is prescribed at nodes of $y=0.5$ and $y=1.5$ boundaries, the essential boundary condition is imposed at nodes of other boundaries) is solved. The results of relative errors of $R_{0}$ and $R_{q}$ by using the ORCM and the CM are shown in Fig. 18 and Fig. 19, respectively. It is seen that the relative errors of $R_{0}$ by using the ORCM are smaller than those by using the CM, the relative errors of $R_{q}$ by using the ORCM are about same as those by using the CM, and the relative errors of both $R_{0}$ and $R_{q}$ become smaller with increase of $n_{t}$.

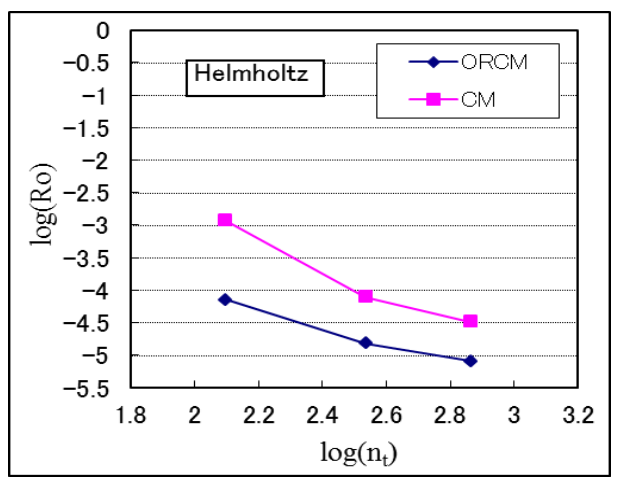

Fig. 18 Relative error $R_{0}$ and convergence for Helmholtz's problem of 3-D.

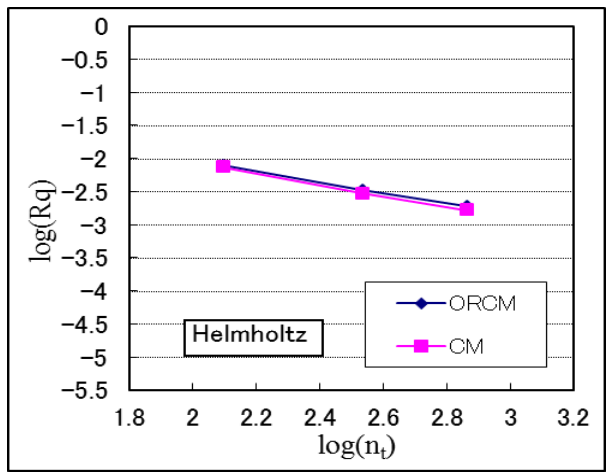

Fig. 19 Relative error $R_{q}$ and convergence for Helmholtz's problem of 3-D.

Figure 20 is a comparison figure of values of $u$ at $x=1, z=1$ by using the ORCM, the CM and the exact solution with nodal model of 1115 nodes ( 729 nodes for the CM) for the first mixed problem. Figure 21 is a comparison figure of values of $\partial u / \partial y$ at $x=1, z=1$ by using the ORCM, the CM and the exact solution with nodal model of 1115 nodes (729 nodes for the CM) for the first mixed problem. Figures 22(a), (b) and (c) are comparison figures of distributions of $\partial u / \partial y$ at $z=1$ by using the ORCM, the CM and the exact solution with nodal model of 1115 nodes (729 nodes for the $\mathrm{CM}$ ) for the first mixed problem. It can be seen that some accurate results of $u$ and $\partial u / \partial y$ are obtained by using both the ORCM and the CM, but the minimum and maximum values of $\partial u / \partial y$ by using the ORCM are more accurate than those by using the CM as shown in Fig. 22.

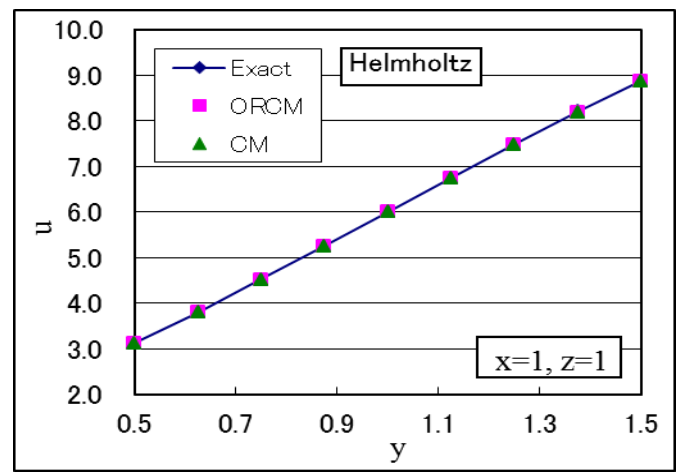

Fig. 20 Values of $u$ at $x=1, z=1$ by nodal model of 1115 nodes ( 729 nodes for CM) for the first mixed problem. 


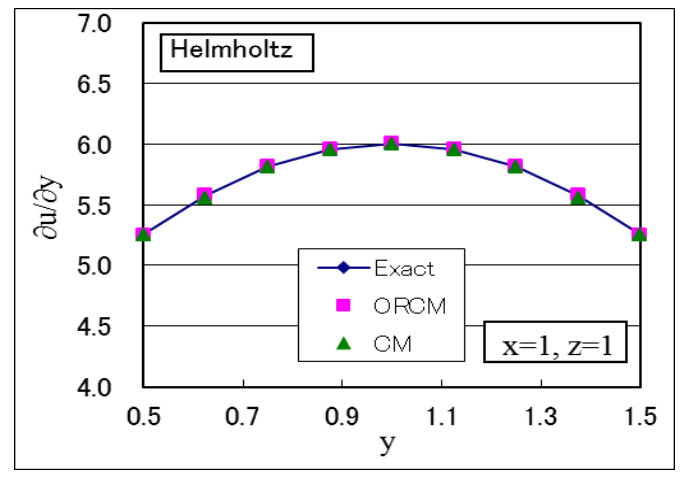

Fig. 21 Values of $\partial u / \partial y$ at $x=1, z=1$ by nodal model of 1115 nodes ( 729 nodes for CM) for the first mixed problem.

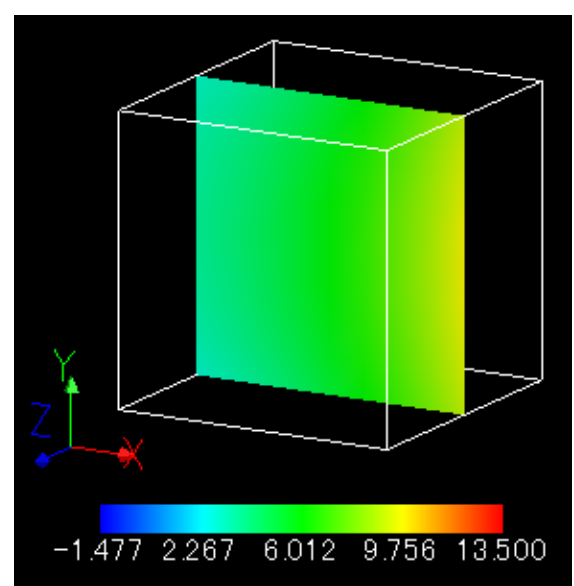

(a) ORCM

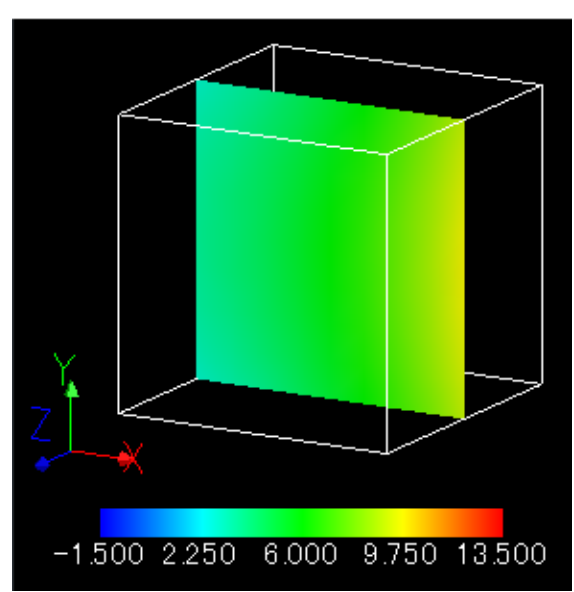

(b) Exact

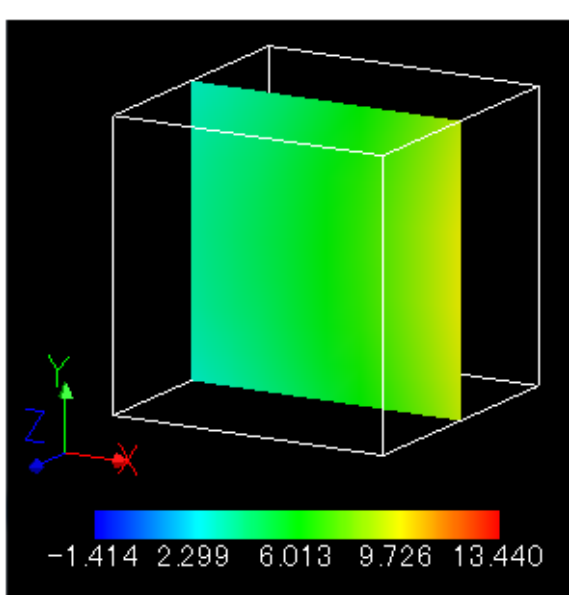

(c) $\mathrm{CM}$

Fig. 22 Values of $\partial \mathrm{u} / \partial \mathrm{y}$ at $z=1$ by nodal model of 1115 nodes ( 729 nodes for $\mathrm{CM})$ for the first mixed problem.

\section{Conclusions}

In this paper, it has been shown that the positivity conditions of boundary points in the ORCM are satisfied and those in the CM are not satisfied by calculated results on the positivity conditions. Two mixed boundary value problems on 2-D Poisson's equation, one mixed boundary value problem on 3-D Poisson's equation and one mixed boundary value problem on 3-D Helmholtz's equation are analyzed by using the ORCM and the CM, respectively. It has been seen that the relative errors by using the ORCM are smaller than those by using the CM, for both the unknown variables and their derivatives of 2-D problems and for the unknown variables of 3-D problems. The relative errors of the 
unknown's derivatives of 3-D problems by using the ORCM are about same as those by using the CM. It has also been shown that the ORCM possesses good accuracy and convergence for both the unknown variables and their derivatives about 2-D problems, and the ORCM possesses good accuracy and convergence for the unknown variables about 3-D problems. The unknown variables and their derivatives by using the ORCM, the CM and the exact solution have also been compared, which show that the ORCM works very well for 2-D boundary value problems, and the ORCM works well like the CM for 3-D boundary value problems.

\section{References}

Aluru, N.R., A point collocation method based on reproducing kernel approximations, International Journal for Numerical Methods in Engineering, Vol.47 (2000), pp.1083-1121.

Atluri, S.N. and Zhu, T., A new meshless local Petrov-Galerkin (MLPG) approach in computational mechanics, Computational Mechanics, Vol.22 (1998), pp.117-127.

Atluri, S.N., Liu, H.T. and Han, Z.D., Meshless local Petrov-Galerkin (MPLG) mixed collocation method for elasticity problems, Computer Modeling in Engineering \& Sciences, Vol.14 (2006a), pp.141-152.

Atluri, S.N., Liu, H.T. and Han, Z.D., Meshless local Petrov-Galerkin (MPLG) mixed finite difference method for solid mechanics, Computer Modeling in Engineering \& Sciences, Vol.15 (2006b), pp.1-16.

Belytschko, T., Lu, Y.Y. and Gu, L., Element free Galerkin methods, International Journal for Numerical Methods in Engineering, Vol.37 (1994), pp.229-256.

Boroomand, B., Tabatabaei, A.A. and Oñate, E., Simple modifications for stabilization of the finite point method, International Journal for Numerical Methods in Engineering, Vol.63 (2005), pp.351-379.

Chen, J.S., Wang, L., Hu, H.Y. and Chi, S.W., Subdomain radial basis collocation method for heterogeneous media, International Journal for Numerical Methods in Engineering, Vol.80 (2009), pp.163-190.

Chi, S.W., Chen, J.S., Hu, H.Y. and Yang, J.P., A gradient reproducing kernel collocation method for boundary value problems, International Journal for Numerical Methods in Engineering, Vol.93 (2013), pp.1381-1402.

Demkowicz, L., Karafilt, A. and Liszka, T., On some convergence results for FDM with irregular mesh, Computer Methods in Applied Mechanics and Engineering, Vol.42 (1984), pp.343-355.

Duarte, C.A. and Oden, J.T., An h-p adaptive method using clouds, Computer Methods in Applied Mechanics and Engineering, Vol.139 (1996), pp.237-262.

Guo, Y.M., An overrange collocation method, Computer Modeling in Engineering \& Sciences, Vol.73 (2011), pp.1-22.

Guo Y.M., Osako, H. and Kamitani, S., Nonlinear analyses by using the ORCM, Journal Computational Science and Technology, Vol.7 (2013), pp.114-125.

$\mathrm{Hu}$, H.Y., Chen, J.S. and Chi, S.W., Perturbation and stability analysis of strong form collocation with reproducing kernel approximation, International Journal for Numerical Methods in Engineering, Vol.88 (2011), pp.157-179.

Jin, X., Li, G. and Aluru, N.R., Positivity conditions in meshless collocation methods, Computer Methods in Applied Mechanics and Engineering, Vol.193 (2004), pp.1171-1202.

Li, S. and Atluri, S.N., The MPLG mixed collocation method for material orientation and topology optimization of anisotropic solids and structures, Computer Modeling in Engineering \& Sciences, Vol.30 (2008), pp.37-56.

Liu, W.K., Jun, S., Li, S., Adee, J. and Belytschko, T., Reproducing kernel particle methods for structural dynamics, International Journal for Numerical Methods in Engineering, Vol.38 (1995), pp.1655-1679.

Melenk, J.M. and Babuska, I., The partition of unity finite element method: basic theory and applications, Computer Methods in Applied Mechanics and Engineering, Vol.139 (1996), pp.289-314.

Nayroles, B., Touzot, G. and Villon, P., Generalizing the FEM: diffuse approximation and diffuse elements, Computational Mechanics, Vol.10 (1992), pp.307-318.

Oñate, E., Idelsohn, S., Zienkiewicz, O.C. and Toylor, R.L., A finite point method in computational mechanics: applications to convective transport and fluid flow, International Journal for Numerical Methods in Engineering, Vol.39 (1996a), pp.3839-3866.

Oñate, E., Idelsohn, S., Zienkiewicz, O.C., Toylor, R.L. and Sacco, C., A stabilized finite point method for analysis of fluid mechanics problems, Computer Methods in Applied Mechanics and Engineering, Vol.139 (1996b), pp. 315-346.

Patanakar, S.V., Numerical Heat Transfer and Fluid Flow (1980), Hemisphere. 
Rajendran, S. and Zhang, B.R., A "FE-meshfree" QUAD4 element based on partition of unity, Computer Methods in Applied Mechanics and Engineering, Vol.197 (2007), pp.128-147.

$\mathrm{Xu}, \mathrm{Q}$., Zhang, Z. and Si, W., Virtual boundary meshless least square collocation method for calculation of 2D multi-domain elastic problems, Engineering Analysis with Boundary Elements, Vol.36 (2012), pp.696-708.

Zhu, T., Zhang, J. and Atluri, S.N., A local boundary integral equation (LBIE) method in computational mechanics and a meshless discretization approach, Computational Mechanics, Vol.22 (1998), pp.223-235. 